\title{
CD30+ Cutaneous T Cell Lymphoma: Response to Rotational Total Skin Electron Irradiation
}

\author{
Hasan H. Danish • Thatcher R. Heumann • Kyle T. Bradley • \\ Jeffrey Switchenko · Natia Esiashvili • Mary Jo Lechowicz • \\ Christopher R. Flowers · Mohammad K. Khan
}

Received: February 25, 2016/ Published online: April 16, 2016

(C) The Author(s) 2016. This article is published with open access at Springerlink.com

\begin{abstract}
Introduction: Rotational total skin electron irradiation (RTSEI) is an effective therapy for cutaneous $\mathrm{T}$ cell lymphoma (CTCL). CD30 expression has been identified as a prognostic factor in CTCL. Therefore, we investigated CD30 status, treatment response, and survival
\end{abstract}

Enhanced content To view enhanced content for this article go to http://www.medengine.com/Redeem/ D4B4F0601BB50AFB.

H. H. Danish · T. R. Heumann · N. Esiashvili ·

M. K. Khan ( $\square)$

Department of Radiation Oncology, Winship

Cancer Institute of Emory University, Atlanta, GA, USA

e-mail: m.k.khan@emory.edu;

drkhurram2000@gmail.com

H. H. Danish

e-mail: hdanish@emory.edu

K. T. Bradley

Department of Pathology and Laboratory Medicine, Emory University School of Medicine, Atlanta, GA, USA

J. Switchenko

Biostatistics Shared Core Resource, Winship Cancer Institute of Emory University, Atlanta, GA, USA

M. J. Lechowicz · C. R. Flowers

Department of Hematology and Medical Oncology, Winship Cancer Institute of Emory University, Atlanta, GA, USA in our cohort of patients with CTCL treated with RTSEI.

Methods: Patients with CTCL treated with RTSEI ( $\geq 30$ Gy) between 2000 and 2013 at our institution were identified, and clinical and pathologic data were retrospectively reviewed. Primary outcomes were complete clinical response (CCR; $>90 \%$ reduction of skin disease burden), relapse-free survival (RFS), and overall survival (OS).

Results: Sixty-eight patients with CTCL treated with RTSEI were identified. Median age at diagnosis was 51 years with median follow-up of 61 months. Median OS was 76 months and median RFS was 11 months. Thirteen patients (19\%) had CD30+ lymphocytes on initial pathology. In the $\mathrm{CD} 30+$ cohort, there were no $\mathrm{T} 2$, eight $\mathrm{T} 3$, and five $\mathrm{T} 4$ cases. In comparison, in the CD30- cohort, there were $18 \mathrm{~T} 2,29 \mathrm{~T} 3$, and $8 \mathrm{~T} 4$ cases $(P=0.01)$. Six weeks post-RTSEI, CCR was $85 \%$ in CD30+ and $81 \%$ in CD30- cases $(P=1)$. Six months post-RTSEI, CCR was $23 \%$ in CD30+ and $50 \%$ in CD30- cases $(P=0.083)$.

Conclusion: RTSEI resulted in excellent CCR at 6 weeks in our cohort of patients with CTCL, with a median RFS of 11 months. We found 
CD30+ patients presented with significantly higher $\mathrm{T}$ stage at time of RTSEI and trended towards decreased CCR at 6 months post-RTSEI compared with the CD30- group.

Keywords: CD30; Complete clinical response; Cutaneous $\mathrm{T}$ cell lymphoma; Mycosis fungoides; Overall survival; Relapse-free survival; Rotational total skin electron irradiation

\section{INTRODUCTION}

Cutaneous $\mathrm{T}$ cell lymphoma (CTCL) is a rare group of lymphoproliferative disorders characterized by localization of $\mathrm{T}$ lymphocytes to the skin with an overall incidence of 10.2 cases per million person-years [1]. It is becoming increasingly recognized that CTCL is heterogeneous, with multiple variants that have unique clinical presentations, histologic features, and therapeutic considerations [2]. The most common subtype of CTCL is mycosis fungoides (MF), which typically presents with pruritic patches and plaques in areas of skin not commonly exposed to sun, and may evolve to cutaneous tumors or erythroderma [3]. Demographic features including African-American race, male gender, and age have been found to be risk factors for CTCL, while extent of skin involvement, overall disease stage, and age are prognostic indicators $[2,3]$. The prognostic significance of lymphocytic CD30 positivity in CTCL prognosis remains controversial: some have reported it as a favorable prognostic factor, [4] while others have shown it to be associated with advanced disease and independently associated with poorer survival [5].

Total skin electron irradiation (TSEI) has been shown to be an effective palliative treatment for CTCL, even following failure with previous treatment modalities. This has been demonstrated in multiple retrospective reviews showing that conventional dose ( $\geq 30$ Gy) TSEI achieves high clinical response rates $[3,6-11]$ typically using large-field/modified Stanford technique (discontinuous irradiation). However, more recent clinical series using dual-field rotational TSEI (RTSEI) technique, where a patient is rotated at a constant speed about the vertical axis while being irradiated with continuous dual-field irradiation (providing a theoretical advantage in dose homogeneity compared to its large-field/modified Stanford counterpart), showed promising results $[12,13]$. Given that there have been no previous studies that have examined the response of CD30+ CTCL to RTSEI, we investigated the effect of CD30 status on treatment response and survival in our cohort of patients with CTCL treated with conventional dose RTSEI.

\section{METHODS}

\section{Patient Population}

After institutional review board (IRB) approval was obtained from Emory University for this study, we retrospectively reviewed medical records of patients treated with RTSEI identified from billing records. Patients were treated between 2000 and 2013 at Emory University, Atlanta, GA, USA. Patient electronic medical records and a previous IRB-approved dermatology database were used to ascertain information regarding demographics, diagnosis, histology, staging, treatment regimens, RTSEI treatment, clinical response, recurrence, and overall survival (OS). Eligibility criteria for the study included: 
histologically confirmed CTCL and a completed first course of conventional dose ( $\geq 30 \mathrm{~Gy}$ ) RTSEI. All patients were staged based on 2007 ISCL EORTC $\mathrm{T}$ and group stage definitions [14].

We identified patients as CD30+ based on initial pathology reports of skin biopsy. All CD30+ patients slides were reviewed by a board-certified hematopathologist (K.B.). Pathologists typically perform CD30 immunohistochemistry when there is concern for transformation with increased numbers of large lymphoid cells. CD30-not-tested cases lacked histological evidence of transformation and thus were grouped for statistical purposes with those that are known to be CD30 negative per pathology report.

\section{Treatment Technique}

RTSEI was administered using a 21EX Varian linear accelerator (LINAC; Varian, Palo Alto, CA, USA) equipped with $6 \mathrm{MeV}$ high-dose total skin electron (HDTSe) mode at a dose rate of $888 \mathrm{MU} / \mathrm{min}$. RTSEI used dual angles field at gantry angles of $241^{\circ}$ and $299^{\circ}$ to cover the upper and the lower halves of the patient body. In the $6 \mathrm{MeV}$ HDTSe mode, our LINAC calibration was $2.99 \mathrm{cGy} / \mathrm{MU}$ at a maximum source to skin distance (SSD) of $100 \mathrm{~cm}$. The patient is placed on a rotating platform at an extended SSD $(315 \mathrm{~cm})$ from the gantry. Both arms are raised overhead and are positioned on the rotator vertical bars. The platform rotates at a constant speed of four revolutions per minute to ensure adequate surface dose buildup.

All patients were treated using a standard method: 36 Gy delivered at 1.5 Gy per fraction, administered three times weekly, typically on a monday-wednesday-friday schedule (4.5 Gy/ week). Eye shields were used upon treatment initiation. Finger/toe nail shields were added after 12 Gy. A one-week mid-treatment break was allowed after delivery of $18 \mathrm{~Gy}$. Optically stimulated luminescent dosimeter (OSLD) measurements at typically under-dosed areas including but not limited to scalp, palms, inner thighs, and bottom of feet were taken prior to the mid-treatment break. Approximately $84 \%$ of patients received additional boost to these under-dosed regions based on clinical assessment of disease response at the end of RTSEI. In total, the total RTSEI regimen required 9 weeks followed by a 2-3-week boost portion.

\section{Covariates}

The overall study cohort was compared across the following covariates: sex, age at diagnosis, age at RTSEI, histology, T stage at start of RTSEI, pre-RTSEI CTCL treatments (therapies received prior to primary RTSEI course including topical, systemic antineoplastic, systemic dermatologic, and phototherapies), maintenance therapy (systemic or topical therapy started within 3-6 months after RTSEI), time from diagnosis to RTSEI, and recurrence.

\section{Outcome Measures}

Primary outcome measures were complete clinical response (CCR) rate, relapse-free survival (RFS), and OS. CCR rate was defined as the proportion of follow-up patients with at least $90 \%$ reduction in cutaneous tumor burden. Patients considered non-complete responders (non-CR) included those with partial response $(>50 \%$ reduction but less than $90 \%$ ), stable disease, recurrence, progression, or death at each of three time points: (1) end of RTSEI treatment, (2) 6 weeks post-RTSEI, and (3) 6 months post-RTSEI. Patients with no previous record of recurrence, those who did not follow-up or had no recorded clinical response at one of the time points were not included in 
the CCR proportion. Response rates were compared across CD30 status and by $\mathrm{T}$ stage using Chi-square or Fisher's exact test, as appropriate.

RFS was calculated as the number of days between start of RTSEI and the date of recurrence. In the case of patients who did not have recurrence, RFS was censored at the date of last follow-up or date of death. OS was the number of days between diagnosis and the date of death or last known follow-up. RFS and OS distributions were estimated using the Kaplan-Meier method. Both RFS and OS were compared across race, T stage, and previously mentioned covariates, using log-rank tests and Cox proportional hazards models. Multivariate Cox models were fit, adjusting for T stage pre-TSEI, and the proportional hazards assumption was checked for all models. The cutoff for statistical significance for all analyses was set at the two-sided alpha error of 0.05. All analysis was done in SAS 9.3 (SAS Institute Inc., Cary, NC, USA).

\section{RESULTS}

\section{Patient Characteristics}

Medical records of 110 patients treated with RTSEI identified from billing records were retrospectively reviewed. After excluding 42 patients who either had a primary diagnosis of leukemia cutis or did not complete conventional dose RTSEI, 68 eligible patients remained. Individual case summaries of all CD30+ patients are shown in Table 1. Patient characteristics of the study cohort, stratified by CD30 status, are summarized in Table 2. Sixty-eight patients were treated with conventional dose RTSEI (range 30-36 Gy). Median age at time of diagnosis was 51 years.
Median follow-up was 61 months. Median time from diagnosis until RTSEI was 35 months. Six patients (46\%) received maintenance therapy following RTSEI. Seven patients (42\%) recurred following RTSEI. Median follow-up time for these patients was 80.2 months.

Pathology confirmed 13 (19\%) CTCL patients as $\mathrm{CD} 30+. \mathrm{CD} 30 \%$ expression ranged from $5 \%$ to $30 \%$ in tumor cells. An example photomicrograph of a $\mathrm{CD} 30+$ transformed CTCL case is shown in Fig. 1. For the CD30+ patients, 7 (54\%) were female and 6 were male (46\%), and 7 were AA (54\%) and 6 were white (46\%). CD30+ patients had a significantly more advanced T stage disease prior to RTSEI (62\% T3, $39 \% \mathrm{~T} 4)$ compared to CD30- patients (33\% T2, $53 \%$ T3, 15\% T4; Table 2). There were no significant differences between CD30 subgroups in regard to sex, race, age at diagnosis, time from diagnosis to RTSEI, treatments prior to RTSEI, maintenance therapy, follow-up time, or proportion that recurred after RTSEI.

\section{Clinical Response Rates}

Table 3 summarizes CCR rates by CD30 status. CD30+ patients had overall CCR rates of $85 \%$ at end of RTSEI, $85 \%$ at 6 weeks follow-up, $23 \%$ at 6 months follow-up. CD30- patients had overall CCR rates of $95 \%$ at the end of RTSEI, $79 \%$ at 6 weeks follow-up, and 50\% at 6 months follow-up. Chi-square and Fisher's exact tests revealed no significant differences in CCR rate between CD30 status subgroups following end of RTSEI $(P=0.24)$ or six weeks $(P=0.44)$, but did trend toward significance at 6 months post-TSEI $(P=0.083)$. This trend was driven by a significantly poorer response at 6 months for CD30+ T3 disease compared to CD30- T3 patients $\quad(P=0.04)$. Further comparisons between CD30 status groups across respective 


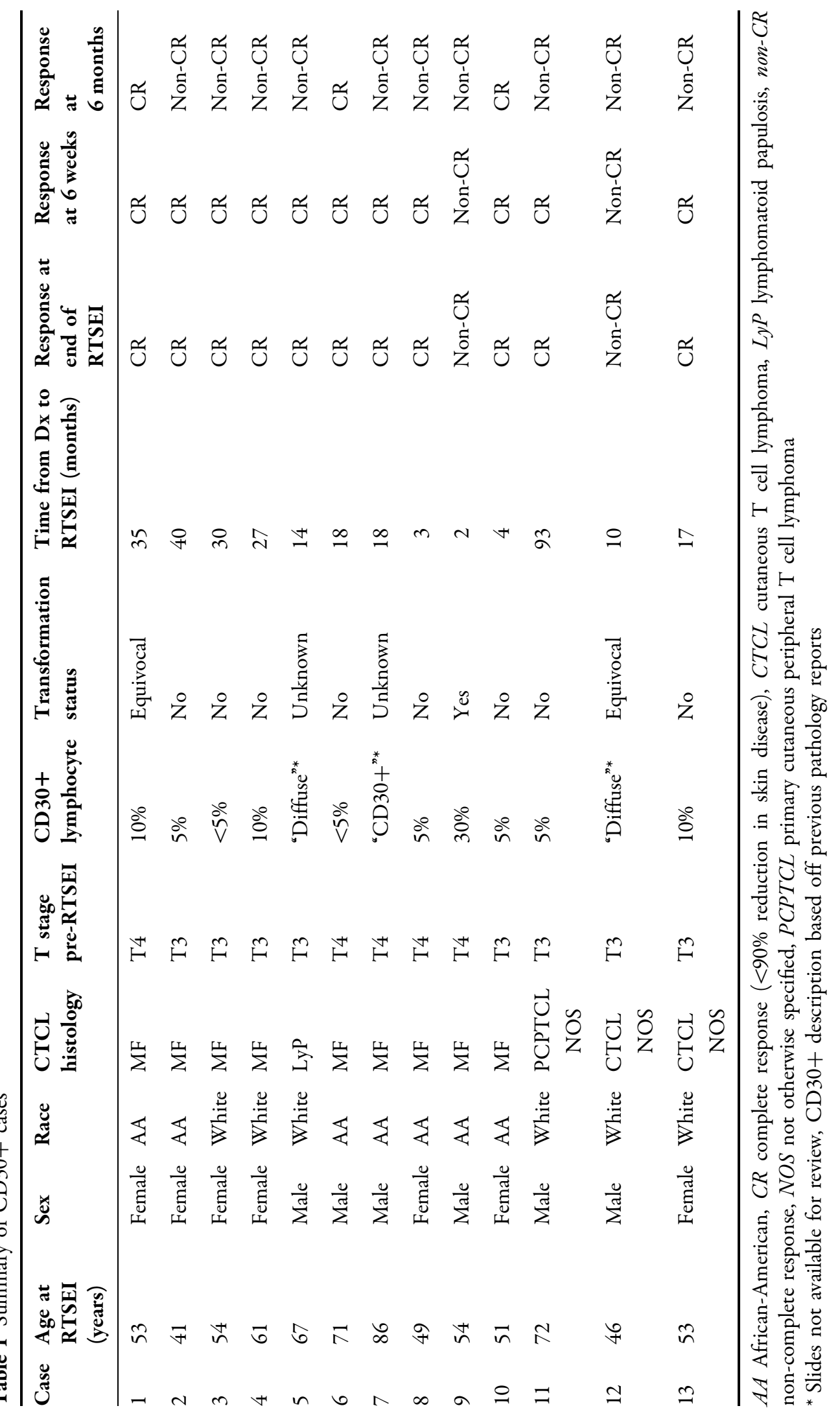


Table 2 Patient characteristics

\begin{tabular}{|c|c|c|c|}
\hline \multirow[t]{2}{*}{ Covariate } & \multicolumn{2}{|l|}{ CD30 status } & \multirow[t]{2}{*}{$P$ value } \\
\hline & $\overline{C D 30-(n=54)}$ & $\mathrm{CD30}+(n=14)$ & \\
\hline \multicolumn{4}{|l|}{ Sex, $n(\%)$} \\
\hline Female & $26(47.3)$ & $7(53.9)$ & 0.67 \\
\hline Male & $29(52.7)$ & $6(46.2)$ & \\
\hline \multicolumn{4}{|l|}{ Race, $n(\%)$} \\
\hline White & $27(50.9)$ & $6(46.2)$ & 0.76 \\
\hline African-American & $26(49.1)$ & $7(53.9)$ & \\
\hline Age, mean & 52.4 & 56.2 & \\
\hline \multicolumn{4}{|c|}{ Time (months) from diagnosis to RTSEI } \\
\hline Median & 16.8 & 35.4 & 0.30 \\
\hline Mean & 27.2 & 35.8 & \\
\hline \multicolumn{4}{|c|}{ Pre-RTSEI tumor $(\mathrm{T})$ stage, $n(\%)$} \\
\hline $\mathrm{T} 2$ & $18(32.8)$ & $0(0.0)$ & 0.01 \\
\hline $\mathrm{T} 3$ & $29(52.7)$ & $8(61.5)$ & \\
\hline $\mathrm{T} 4$ & $8(14.6)$ & $5(38.5)$ & \\
\hline \multicolumn{4}{|c|}{ Pre-RTSEI CTCL treatment } \\
\hline \multicolumn{4}{|c|}{ Previous use of topical agents, $n(\%)$} \\
\hline No & $25(45.5)$ & $5(38.5)$ & 0.65 \\
\hline Yes & $30(54.6)$ & $8(61.5)$ & \\
\hline \multicolumn{4}{|c|}{ Previous use of systemic antineoplastic agents, $n(\%)$} \\
\hline No & $27(49.1)$ & $6(46.2)$ & 0.85 \\
\hline Yes & $28(50.9)$ & $7(53.9)$ & \\
\hline \multicolumn{4}{|c|}{ Previous use of systemic dermatologic agents, $n$ (\%) } \\
\hline No & $43(78.2)$ & $12(92.3)$ & 0.44 \\
\hline Yes & $12(21.8)$ & $1(7.7)$ & \\
\hline \multicolumn{4}{|c|}{ Previous use of phototherapy, $n(\%)$} \\
\hline No & $29(52.7)$ & $6(46.2)$ & 0.67 \\
\hline Yes & $26(47.3)$ & $7(53.8)$ & \\
\hline \multicolumn{4}{|c|}{ Maintenance therapy post-RTSEI, $n$ (\%) } \\
\hline No & $35(63.6)$ & $7(53.9)$ & 0.54 \\
\hline Yes & $20(36.4)$ & $6(57.1)$ & \\
\hline
\end{tabular}


Table 2 continued

\begin{tabular}{llll}
\hline Covariate & CD30 status & & P value \\
\cline { 2 - 3 } & CD30- $(n=54)$ & CD30+ $(\boldsymbol{n}=\mathbf{1 4})$ \\
\hline Recurrence following RTSEI, $n(\%)$ & & \\
No & $18(32.7)$ & $6(46.2)$ & 0.42 \\
Yes & $37(67.3)$ & $7(53.9)$ & \\
\hline
\end{tabular}

CTCL cutaneous T cell lymphoma, RTSEI rotational total skin electron irradiation

Bold $P$ values are statistically significant $(P<0.05)$

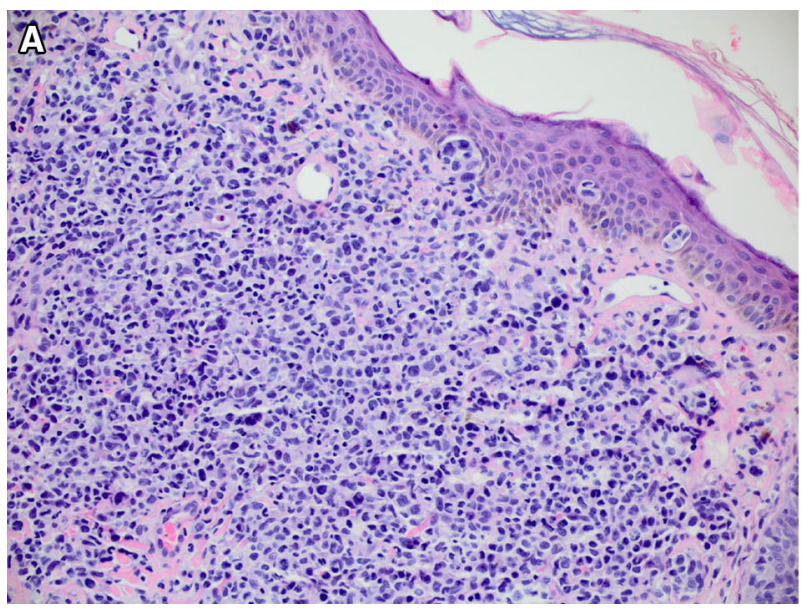

Fig. 1 Transformed cutaneous T cell lymphoma (Case \#9 from Table 1). a Large transformed lymphoma cells with focal epidermotropism (H\&E stain, original magnification

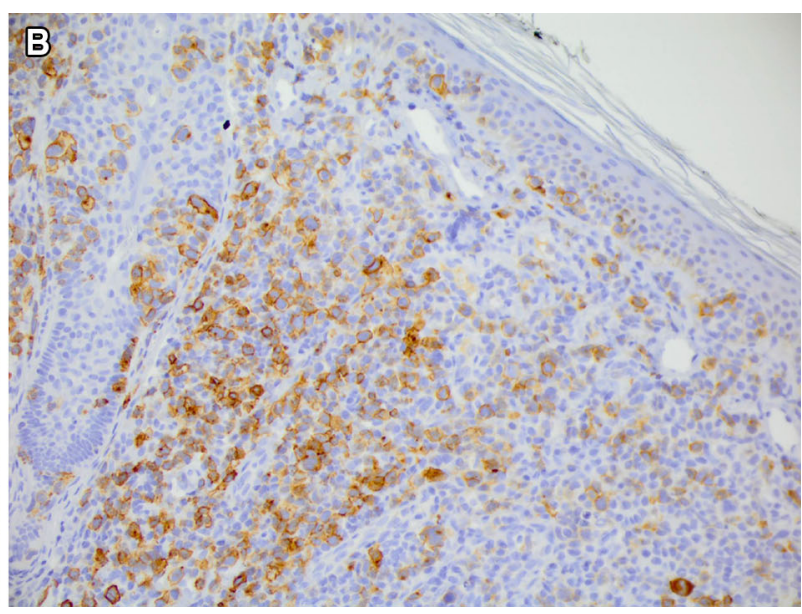

$\times 200)$. b Approximately $30 \%$ of lymphoma cells are positive for CD30 (original magnification $\times 200$ )

Table 3 Complete clinical response rates ( $>90 \%$ reduction in tumor burden), stratified by $\mathrm{T}$ stage pre-RTSEI and CD30 status

\begin{tabular}{|c|c|c|c|c|c|c|c|c|c|}
\hline \multirow{4}{*}{$\begin{array}{l}\text { T stage } \\
\text { pre-RTSEI }\end{array}$} & \multicolumn{9}{|c|}{ Follow-up period } \\
\hline & \multicolumn{3}{|c|}{ End of RTSEI } & \multicolumn{3}{|c|}{ Six weeks post-RTSEI } & \multicolumn{3}{|c|}{ Six months post-RTSEI } \\
\hline & \multicolumn{3}{|c|}{ CD30 status } & \multicolumn{3}{|c|}{ CD30 status } & \multicolumn{3}{|c|}{ CD30 status } \\
\hline & $\begin{array}{l}\text { CD30+ } \\
n(\%)\end{array}$ & $\begin{array}{l}\text { CD30- } \\
n(\%)\end{array}$ & $P$ value & $\begin{array}{l}\text { CD30+ } \\
n(\%)\end{array}$ & $\begin{array}{l}\text { CD30- } \\
n(\%)\end{array}$ & $P$ value & $\begin{array}{l}\text { CD30+ } \\
n(\%)\end{array}$ & $\begin{array}{l}\text { CD30- } \\
n(\%)\end{array}$ & $P$ value \\
\hline All $T$ stages & $11(85)$ & $52(95)$ & 0.24 & $11(85)$ & $42(79)$ & 1 & $3(23)$ & $24(50)$ & 0.083 \\
\hline $\mathrm{T} 2$ & - & $18(100)$ & - & - & $15(88)$ & - & - & $5(42)$ & - \\
\hline $\mathrm{T} 3$ & $7(88)$ & $27(93)$ & 0.53 & $7(88)$ & $22(79)$ & 1 & $1(13)$ & $16(57)$ & 0.044 \\
\hline T4 & $4(80)$ & $7(88)$ & 1 & $4(80)$ & $5(62.5)$ & 1 & $2(40)$ & $3(38)$ & 1 \\
\hline
\end{tabular}

Bold $P$ values are statistically significant $(P<0.05)$ 
T stages at all other follow-up time points showed no significance difference in CCR rates.

\section{Relapse-Free Survival}

As seen in Table 4, CD30+ patients had a median RFS of 12.1 months (8.3 and 13.2 for $\mathrm{T} 3$ and $\mathrm{T} 4$ disease, respectively, not significantly different). CD30- patients had a median RFS of 9.9 months $(14.3,11.3$, and 6.0 months for T2, $\mathrm{T} 3$, and T4 disease, respectively, with no significant difference between stages T3 and T4). Kaplan-Meier RFS curves comparing CD30 groups and $\mathrm{CD} 30+$ patients by $\mathrm{T}$ stage are shown in Fig. 2.

On multivariate analysis, controlling for T stage, CD30 status was not associated with RFS $(P=0.75)$. Maintenance therapy following RTSEI was associated with significantly improved RFS in CD30- group [hazard ratio $(\mathrm{HR})=2.79 ; \quad 95 \%$ confidence interval $(\mathrm{CI})$ 1.17-6.66; $P=0.021]$. This association was not observed in the CD30+ group $(P=0.383)$. All other covariates such as race, sex, T stage, histology, age at diagnosis, time from diagnosis to start of TSEI, and pre-RSTEI treatment did not have a clinically significant association with RFS on univariate or multivariate analyses in either $\mathrm{CD} 30+$ or CD30- groups.

\section{Overall Survival}

As seen in Table 4, CD30+ patients had a median OS of 103.3 months overall with no significant difference in OS between stages T3 and T4 $(P=0.65)$. CD30- patients had a median OS of 74.5 months with a trend towards poorer survival for T4 compared to T3 patients (103.3 months for T3, 30.5 months for T4, $P=0.09)$. There was no significant difference in OS across CD30 status $(P=0.57)$, T3 $(P=0.95)$, or T4 $(P=0.10)$ subgroups. Kaplan-Meier OS curves for comparing CD30 groups and $\mathrm{CD} 30+$ patients by $\mathrm{T}$ stage are shown in Fig. 2.

On univariate analysis, CD30 status was not associated with OS $(P=0.57)$. In the CD30+ group, non-MF histology (compared to MF histology) was associated with poorer OS in univariate analysis $(\mathrm{HR}=10.65 ; \quad P=0.025)$. However, after controlling for T stage pre-RTSEI, this observed effect only trended towards significance $(P=0.080)$. In the CD30group, lower T stage $(\mathrm{HR}=0.26 ; P=0.049)$ in univariate analysis was associated with

Table 4 RFS and OS, stratified by T stage pre-RTSEI and CD30 status

\begin{tabular}{|c|c|c|c|c|c|c|}
\hline \multirow[t]{2}{*}{ T stage pre-RTSEI } & \multicolumn{3}{|c|}{ Median RFS (months) } & \multicolumn{3}{|c|}{ Median OS (months) } \\
\hline & CD30- & CD30+ & $P$ value & $\overline{\text { CD30- }}$ & CD30+ & $P$ value \\
\hline All $\mathrm{T}$ stages & 9.9 & 12.1 & 0.75 & 74.5 & 103.3 & 0.57 \\
\hline $\mathrm{T} 2$ & 14.3 & - & - & - & - & - \\
\hline T3 & 11.3 & 8.3 & 0.64 & 103.3 & 90.7 & 0.95 \\
\hline $\mathrm{T} 4$ & 6.0 & 13.2 & 0.51 & 30.5 & - & 0.10 \\
\hline
\end{tabular}

OS overall survival, RFS relapse-free survival, RTSEI rotational total skin electron irradiation 


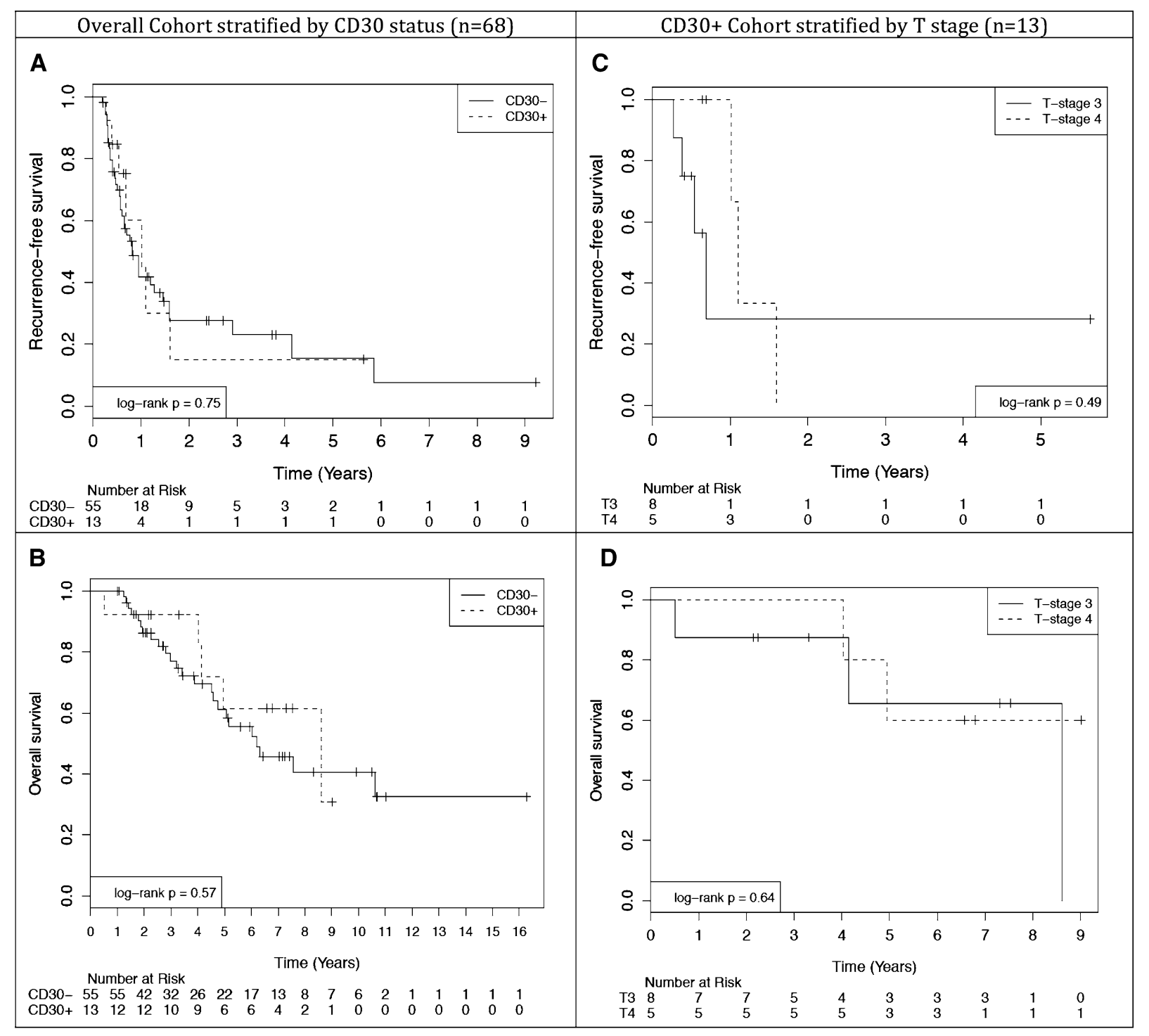

Fig. 2 Survival outcomes. a RFS comparing CD30+ vs. CD30 - patients. b OS comparing CD30+ vs. CD30patients. c RFS for CD30+ patients comparing stage T3 and T4 pre-RTSEI. d OS for CD30+ patients comparing

stage T3 and T4 pre-RTSEI. OS overall survival, RFS relapse-free survival, RTSEI rotational total skin electron irradiation

improved OS. In multivariate analysis, increased time from diagnosis to RTSEI $(\mathrm{HR}=0.98, P=0.017)$ and younger age at RTSEI $(\mathrm{HR}=1.06 ; P=0.003)$ were associated with a significantly improved OS. Maintenance

therapy failed to show any significant improvement in OS in both CD30+ $(P=0.998) \quad$ and $\quad \mathrm{CD} 30-\quad(P=0.242) \quad$ in multivariate analysis controlling for $\mathrm{T}$ stage pre-RTSEI. 


\section{DISCUSSION}

At present there in no long-term curative treatment for CTCL. Management is difficult as there are no standardized treatment regimens, there is prognostic heterogeneity even within group stages, and high-quality trials are lacking due to disease rarity. There is an urgent need to identify patients at high or low risk for disease progression or mortality to aid in clinical decisions about treatment including escalation/de-escalation of radiation dose or initiation/holding of systemic agents. Since treatment for CTCL, especially advanced-stage disease, is palliative, improved prognostication would help avoid treatment toxicity in cases at low risk for progression. Recently, the prognostic Cutaneous Lymphoma International Prognostic index (CLIPi) was developed for early- and late-stage MF using a cohort of 1502 UK patients and validated in a set of 1221 patients treated at MD Anderson Cancer Center (MDACC; Houston, TX, USA); however, this has yet to be validated in a prospective manner [15-17]. The Cutaneous Lymphoma International Consortium (CLIC) developed a prognostic score for advanced MF stage IIB to IV and Sezary syndrome (SS) with 1394 patients from 29 centers worldwide including the following factors: stage IV disease, age greater than 60 years, large cell transformation, and elevated serum lactate dehydrogenase [18].

CD30 is a member of the tumor necrosis receptor family, and is found on activated $\mathrm{T}$ and B cells. A recent study of 47 non-transformed MF cases from the University of Pittsburgh (Pittsburgh, PA, USA) reported that patients were found to have worse survival, higher stage at diagnosis, and higher maximum stage if dermal cells are CD30+ (>4.7\%) [5]. A study of 51 patients with large cell transformation of
MF at Memorial Sloan Kettering Cancer Center (New York, NY, USA) reported that predominance of CD30 in the epidermis rather than dermis was associated with poorer survival [19]. However, a retrospective analysis of 100 patients with transformed MF from the Netherlands showed that CD30 negativity was associated with multivariate analysis with reduced disease-specific survival (DSS) and OS [4]. In a long-term outcomes study, CD30 was found to have no significant effect on OS, DSS, and progression-free survival (PFS) in $1263 \mathrm{MF}$ SS patients at MDACC [17]. The CLIC reported that when CD30 is scored positive above $10 \%$ of tumoral cells, it is not statistically significant for OS but is associated with worse OS in the subset of patients with T3 disease [18]. Finally, CD30 is also targetable with brentuximab vedotin, a monoclonal chimeric antibody conjugated to antitubulin agent monomethyl auristatin E. A recent phase 2 study found that brentuximab vedotin has significant objective global response $70 \%(21 / 30)$ in treatment patients with refractory or advanced MF/SS who have a range of CD30 expression levels [20]. Similarly, another phase 2 trial found overall response of $73 \%(35 / 48)$ in patients with CD30+ lymphoproliferative disorders treated with brentuximab vedotin [21].

Based on the varying reports of the prognostic significance of CD30, we sought to assess the response and survival of our cohort of patients treated with RTSEI. We found 13 of 68 patients were CD30+ (range 5\% to 30\%); they presented with significantly higher $\mathrm{T}$ stage at time of RTSEI and trended towards decreased CCR at 6 months post-RTSEI compared with the CD30 - group. One patient in our cohort had $1 \%$ CD30+ lymphocytes on pathology and was classified as negative for CD30; however, when this patient was added to the $\mathrm{CD} 30+$ cohort (data not reported in results) the CCR was 
significantly reduced at 6 months $(P=0.05)$. Regardless, the difference in 6 month CCR was primarily driven by those with $\mathrm{T} 3$ stage disease. Overall, CD30+ patients had excellent CCR rates of $85 \%$ at end of RTSEI but most had progressive disease at 6 months. Maintenance therapy including topical, systemic antineoplastic, systemic dermatologic, and phototherapies failed to show any significant improvement in RFS in the CD30+ group. Additionally, maintenance therapy failed to show any significant improvement in OS in both CD30+ and CD30- groups. Although direct comparison is not possible without further study, our rates of clinical response are comparable to those recently reported for brentuximab vedotin.

Additionally, CD30+ patients may benefit from the addition of novel targeted agents such as Brentuximab vedotin to radiation, perhaps concurrently or sequentially. Recently, the concept of combined radiation and immunotherapy to enhance clinical outcomes has advanced. To date there is no trial of the safety of brentuximab vedotin with radiation. We feel prospective trial development of combined brentuximab vedotin and radiation for CTCL should proceed based on the results of recent phase 2 trials.

Our analysis is limited by small sample size; however, this is the largest report of CD30+ patients, and their response to radiotherapy. More specifically, the small sample size precluded us from examining in more detail the role of maintenance systemic and dermatologic agents. In addition, if a patient did not follow up or did not have a recorded clinical response at the specified time points without record of recurrence by other providers, they were excluded from CCR analysis which further decreased patient numbers in the present analysis. Additionally, as this was a retrospective review, many patients had incomplete clinicopathological information, information from outside hospitals, or were lost to follow-up. Incomplete data regarding CD30 status increases confounding as patients deemed by clinicians to have aggressive disease or transformation often have more thorough workup. Strengths of our study included documentation of clinical response across multiple post-treatment time points, single institution experience with standardized RTSEI delivery and dosing, and all CD30+ cases were reviewed by a hematopathologist to confirm CD30 status.

\section{CONCLUSIONS}

In this retrospective study, RTSEI for CTCL resulted in excellent CCR at 6 weeks, with a median RFS of 11 months. This is consistent with and improves upon treatment results reported previously. CD30+ patients receiving RTSEI were found to have higher $\mathrm{T}$ stage and trended towards decreased CCR at 6 months post-RTSEI compared with the CD30- patients. Maintenance therapy including topical, systemic antineoplastic, systemic dermatologic, and phototherapies failed to show any significant improvement in RFS in the CD30+ group. Future work should examine quality of life metrics, the role of maintenance systemic or targeted treatments, and combination RTSEI and brentuximab vedotin in CD30+ patients with CTCL.

\section{ACKNOWLEDGMENTS}

No funding or sponsorship was received for this study. The article processing charges for this publication were funded by the authors. All named authors meet the International 
Committee of Medical Journal Editors (ICMJE) criteria for authorship of this manuscript, take responsibility for the integrity of the work as a whole, and have given final approval for the version to be published.

Disclosures. Hasan H. Danish, Thatcher R. Heumann, Kyle T. Bradley, Jeffrey Switchenko, Natia Esiashvili, Mary Jo Lechowicz, Christopher R. Flowers, and Mohammad K. Khan have nothing to disclose.

\section{Compliance}

with

Ethics

Guidelines. Institutional review board (IRB) approval was obtained from Emory University for this study

Open Access. This article is distributed under the terms of the Creative Commons AttributionNonCommercial 4.0 International License (http://creativecommons.org/licenses/by-nc/4. $0 /$ ), which permits any noncommercial use, distribution, and reproduction in any medium, provided you give appropriate credit to the original author(s) and the source, provide a link to the Creative Commons license, and indicate if changes were made.

\section{REFERENCES}

1. Korgavkar K, Xiong $\mathrm{M}$, Weinstock M. Changing incidence trends of cutaneous T-cell lymphoma. JAMA dermatology. 2013;149(11):1295-9.

2. Imam MH, Shenoy PJ, Flowers CR, Phillips A, Lechowicz MJ. Incidence and survival patterns of cutaneous T-cell lymphomas in the United States. Leuk Lymphoma. 2013;54(4):752-9.

3. Navi D, Riaz N, Levin YS, Sullivan NC, Kim YH, Hoppe RT. The Stanford University experience with conventional-dose, total skin electron-beam therapy in the treatment of generalized patch or plaque (T2) and tumor (T3) mycosis fungoides. Arch Dermatol. 2011;147(5):561-7.
4. Benner MF, Jansen PM, Vermeer MH, Willemze R. Prognostic factors in transformed mycosis fungoides: a retrospective analysis of 100 cases. Blood. 2012;119(7):1643-9.

5. Edinger JT, Clark BZ, Pucevich BE, Geskin LJ, Swerdlow SH. CD30 expression and proliferative fraction in nontransformed mycosis fungoides. Am J Surg Pathol. 2009;33(12):1860-8.

6. Morris SL, McGovern M, Bayne S, Wain M, Child F, Whittaker S. Results of a 5-week schedule of modern total skin electron beam radiation therapy. Int J Radiat Oncol Biol Phys. 2013;86(5):936-41.

7. Parida DK, Verma KK, Chander S, Joshi RC, Rath GK. Total skin electron irradiation therapy in mycosis fungoides using high-dose rate mode: a preliminary experience. Int $\mathrm{J}$ Dermatol. 2005;44(10):828-30.

8. Parida DK, Verma KK, Rath GK. Total skin electron irradiation treatment for mycosis fungoides with a new alternate daily treatment schedule to minimize radiation-associated toxicity: a preliminary experience. Clin Exp Dermatol. 2009;34(5):e37-9.

9. Wagner AE, Wada D, Bowen G, Gaffney DK. Mycosis fungoides: the addition of concurrent and adjuvant interferon to total skin electron beam therapy. Br J Dermatol. 2013;169(3):715-8.

10. Ysebaert L, Truc G, Dalac S, Lambert D, Petrella T, Barillot I, et al. Ultimate results of radiation therapy for T1-T2 mycosis fungoides (including reirradiation). Int $\mathrm{J}$ Radiat Oncol Biol Phys. 2004;58(4):1128-34.

11. Freeman CR, Suissa S, Shenouda G, Vuong T, Souhami L, Pla M, et al. Clinical experience with a single field rotational total skin electron irradiation technique for cutaneous T-cell lymphoma. Radiother Oncol. 1992;24(3):155-62.

12. Heumann TR, Esiashvili N, Parker S, Switchenko JM, Dhabbaan A, Goodman M, et al. Total skin electron therapy for cutaneous T-cell lymphoma using a modern dual-field rotational technique. Int J Radiat Oncol Biol Phys. 2015;92(1):183-91.

13. Kumar PP, Good RR, Jones EO, McAnulty BE, Reeves MA. Dual-field rotational (DFR) technique for total-skin electron-beam therapy (TSEBT). Am J Clin Oncol. 1987;10(4):344-54.

14. Olsen E, Vonderheid E, Pimpinelli N, Willemze R, Kim Y, Knobler R, et al. Revisions to the staging and classification of mycosis fungoides and Sezary syndrome: a proposal of the International Society for Cutaneous Lymphomas (ISCL) and the cutaneous lymphoma task force of the European 
Organization of Research and Treatment of Cancer (EORTC). Blood. 2007;110(6):1713-22.

15. Agar NS, Wedgeworth E, Crichton S, Mitchell TJ, Cox M, Ferreira $S$, et al. Survival outcomes and prognostic factors in mycosis fungoides/Sezary syndrome: validation of the revised International Society for Cutaneous Lymphomas/European Organisation for Research and Treatment of Cancer staging proposal. J Clin Oncol. 2010;28(31):4730-9.

16. Benton EC, Crichton S, Talpur R, Agar NS, Fields PA, Wedgeworth E, et al. A cutaneous lymphoma international prognostic index (CLIPi) for mycosis fungoides and Sezary syndrome. Eur J Cancer. 2013;49(13):2859-68.

17. Talpur R, Singh L, Daulat S, Liu P, Seyfer S, Trynosky $\mathrm{T}$, et al. Long-term outcomes of 1,263 patients with mycosis fungoides and Sezary syndrome from 1982 to 2009. Clinical Cancer Res. 2012;18(18):5051-60.

18. Scarisbrick JJ, Prince HM, Vermeer MH, Quaglino P, Horwitz S, Porcu P, et al. Cutaneous lymphoma international consortium study of outcome in advanced stages of mycosis fungoides and Sezary syndrome: effect of specific prognostic markers on survival and development of a prognostic model. J Clin Oncol. 2015;33(32):3766-73.

19. Pulitzer M, Myskowski PL, Horwitz SM, Querfeld C, Connolly B, Li J, et al. Mycosis fungoides with large cell transformation: clinicopathological features and prognostic factors. Pathology. 2014;46(7): 610-6.

20. Kim YH, Tavallaee M, Sundram U, Salva KA, Wood GS, Li S, et al. Phase II investigator-initiated study of brentuximab vedotin in mycosis fungoides and Sezary syndrome with variable CD30 expression level: a multi-institution collaborative project. J Clin Oncol. 2015;33(32):3750-8.

21. Duvic M, Tetzlaff MT, Gangar P, Clos AL, Sui D, Talpur R. Results of a phase II trial of brentuximab vedotin for CD30+ cutaneous T-cell lymphoma and lymphomatoid papulosis. J Clin Oncol. 2015; 33(32):3759-65. 